\title{
Analysis of arrays of printed strip dipole antennas and broadband antennas of six series fed strip dipoles
}

\author{
Arianit Maraj ${ }^{1}$, Ruzhdi Sefa ${ }^{2}$ \\ ${ }^{1}$ Faculty of Computer Science, Public University of Prizren, Prizren, Republic of Kosova \\ ${ }^{2}$ Faculty of Electrical and Computer Engineering, University of Prishtina, Prishtina, Republic of Kosova \\ Email address: \\ arianit.maraj@uni-prizren.com (A. Maraj),ruzhdi.sefa@uni-pr.edu (R. Sefa)
}

To cite this article:

Arianit Maraj, Ruzhdi Sefa. Analysis of Arrays of Printed Strip Dipole Antennas and Broadband Antennas of Six Series Fed Strip Dipoles. International Journal of Wireless Communications and Mobile Computing. Vol. 1, No. 3, 2013, pp. 74-81.

doi: $10.11648 /$ j.wcmc.20130103.11

\begin{abstract}
Dipole antennas are suitable in wireless multi-band technology, because they are simple to design and have excellent radiation properties. Also, it is well known that analysis of series-fed strip dipoles antennas are very hard to do because of the presence of dielectric inhomogeneity. In addition, the strip dipoles are closely spaced and connected through transmission lines that are integral part of the antenna. Therefore, an analysis method that takes into account the presence of dielectric substrate and the effect of connecting lines is presented to accurately design these antennas. In this paper, arrays of printed strip dipoles antennas are analyzed based on moment method in the spectral domain. The double-sided configuration has been selected because it offers several practical advantages. In this paper, further we will complicate the analysis by taking into account broadband antennas comprised of six series fed strip dipoles.
\end{abstract}

Keywords: Dipole Antennas, Moment Method, Stripline, Double-Sided Configuration

\section{Introduction}

Printed dipole antennas are commonly used as radiating elements in wireless communications as they are easy to fabricate, have low cost, and high performance. The antennas are comprised of a number of closely spaced narrow conductive strips printed on electrically thin dielectric substrates. Although the antennas are easy to make and inexpensive, they posses tremendous performance capabilities including high gain, dual polarization, broadband or multi-band operation [1-4]. In addition when these antennas are fed in series they generally produce endfire radiation making them suitable for use with plane or shaped reflectors, a configuration commonly employed in base stations of wireless communications systems [5].

Printed dipole radiators have been popular candidates for phased array antennas that contain many elements because of their suitability for integration with microwave integrated circuit modules [6-8]. These antennas have also been considered for application in far-infrared and millimeter-wave imaging systems [9, 10].

Although printed strip antennas are intrinsically simple, the presence of the dielectric inhomogeneity presents a significant challenge in finding computationally reasonable CAD tool for designing these antennas.

Single strip dipoles printed on electrically thin, low permittivity substrates behave similarly to ordinary dipoles, and they have often been designed based on experimental trial and error $[11,12]$. However, when dipoles of different lengths are closely spaced and/or connected with each other with transmission lines, the radiation characteristics depend on a large number of design parameters. An analysis method that can handle three-dimensional structures containing both dielectric and conducting materials should be used to correctly analyze these antennas. However such an analysis is computationally intensive and it provides no easy way to select optimal design parameters. A more efficient full wave analysis would be to consider dipoles printed on an infinite substrate, and apply a moment method in the spectral domain. Such an approach is used here to analyze strip dipoles printed on a thin ungrounded dielectric substrate. Similar techniques have been employed for the analysis of strip dipoles printed on a grounded dielectric substrate [13] and on a dielectric halfspace [10].

The procedure for analyzing arrays of printed strip dipoles antennas based a moment method in the spectral 
domain is presented. The dipoles can be connected to each other.

\section{Antenna Configuration}

Fig. 1 is a schematic drawing of an array of series-fed printed strip dipoles. The antenna consists of several printed strip dipoles of different lengths, with the arms printed on opposite sides of an electrically thin dielectric substrate that are connected through parallel striplines.

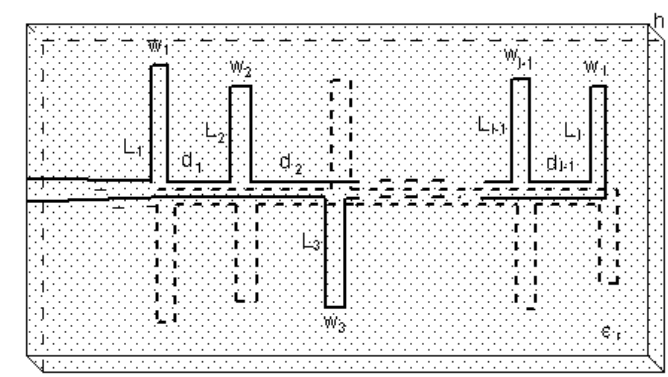

Figure1. Drawing of a series-fed printed strip dipole antenna

The parallel stripline consists of two broadside-coupled strips; each section may have different widths, and is characterized its characteristic impedance $Z_{0}$ and effective relative permittivity $\varepsilon_{\text {eff }}$. The antenna is fed from and the other end is open circuited. In general, some strip dipoles may be parasitically placed and several dipoles can be fed simultaneously. Usually a microstrip-to-parallel stripline transition is used to feed the antenna from a conventional coaxial connector [14]. The double-sided configuration has been selected because it offers several practical advantages such as: when required the line polarity between the strip dipoles can be easily reversed as shown in Fig. 1, parallel striplines offer low as well as large values of line impedances with practically reasonable conductor widths, and parallel striplines do not radiate since they are closely spaced.

\section{Analysis Model}

Analyses of series-fed printed strip dipole antennas are complicated by the presence of the dielectric inhomogeneity. In addition, the strip dipoles are closely spaced and connected through transmission lines that are integral part of the antenna. Since they are printed on finite dielectric substrates, they represent three-dimensional composite structures consisting of conducting and dielectric parts. A space-domain moment method analysis would require solving for the conducting currents on the strips and the polarization currents in the dielectric volume, resulting in a large number of unknowns [15]. To simplify the analysis, it is assumed that the conductive strips are printed on an infinite dielectric substrate and the effect of the connecting line is accounted for separately by using a standard transmission line theory. The approach used to analyze the antennas is described in the following.

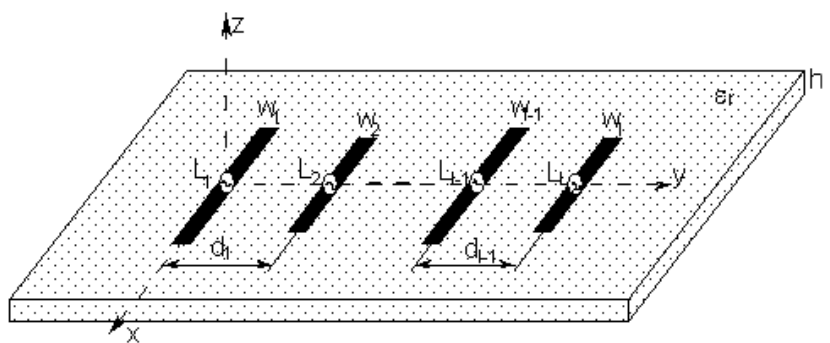

Figure2. Geometry of a group of l parallel printed strip dipoles.

Fig. 2 shows the geometry of $l$ strip dipoles printed on a dielectric substrate of height $h$ and relative permittivity $\varepsilon_{r}$. The strips have dimensions $L_{1}, w_{1}, L_{2}, w_{2}, \ldots, L_{l}, w_{l}$ and are separated by distances $d_{1}, d_{2}, \ldots, d_{l}$. They are assumed to be thin $\left(w_{i}<<L_{i}, i=1,2, \ldots, l\right)$ so only the $x$ directed currents are considered. All the strip dipoles that are connected with transmission lines are assumed to be fed by delta gap generators. The procedure is similar to that used for analysis of microstrip dipoles [13]. The $x$ component of the electric field on the strip side of the dielectric substrate due to strip currents can be expressed as:

$$
E_{x}(x, y, 0)=\frac{1}{4 \pi^{2}} \int_{-\infty}^{\infty} \int_{-\infty}^{\infty} G_{x x}\left(k_{x}, k_{y}\right) J_{x}\left(k_{x}, k_{y}\right) e^{j\left(k_{x} x+k_{y} y\right)} d k_{x} d k_{y}
$$

where $J_{x}\left(k_{x}, k_{y}\right)$ is the Fourier transform of the surface current density on the strips, $j_{x}(x, y)$, and $G_{x x}\left(k_{x}, k_{y}\right)$ is the spectral domain Green's function given as:

$$
G_{x x}\left(k_{x}, k_{y}\right)=-\frac{Z_{a}}{k_{0} \beta^{2}}\left[\frac{k_{x}^{2} k_{1} k_{2}}{T_{m}}\left(\varepsilon_{r} k_{2} \cos k_{1} h+j k_{1} \sin k_{1} h\right)+\frac{k_{0}^{2} k_{y}^{2}}{T_{e}}\left(k_{1} \cos k_{1} h+j k_{2} \sin k_{1} h\right)\right]
$$

$T_{m}=2\left(\varepsilon_{r} k_{2} \cos k_{1} h / 2+j k_{1} \sin k_{1} h / 2\right)\left(k_{1} \cos k_{1} h / 2+j \varepsilon_{r} k_{2} \sin k_{1} h / 2\right)(3)$

$$
T_{e}=2\left(k_{2} \cos k_{1} h / 2+j k_{1} \sin k_{1} h / 2\right)\left(k_{1} \cos k_{1} h / 2+j k_{2} \sin k_{1} h / 2\right)
$$

Where $k_{1}^{2}=\varepsilon_{r} k_{0}^{2}-\beta^{2}, \quad \operatorname{Im}\left(k_{1}\right)<0, k_{2}^{2}=k_{0}^{2}-\beta^{2}, \quad \operatorname{Im}\left(k_{2}\right)<0$, $\beta^{2}=k_{x}^{2}+k_{y}^{2}, k_{0}=\omega \sqrt{\varepsilon_{0} \mu_{0}}$, and $Z_{a}=\sqrt{\mu_{0} / \varepsilon_{0}}$.

An electric field integral equation is formed by requiring that the total electric field, the scattered electric field of Eq. (1) plus the incident field be zero on the strip surfaces. In order to solve this equation, first the unknown current density on the strips is expanded in a set of $N=N_{1}+N_{2}+\ldots+N_{l}$ basic functions with unknown coefficients $I_{n}$ as:

$$
\begin{gathered}
j_{x}(x, y)=\sum_{n=1}^{N} I_{n} f_{n}(x, y) \\
f_{n}(x, y)=\frac{1}{\pi \sqrt{\left(w_{i} / 2\right)^{2}+\left(y-y_{n}\right)^{2}}} \frac{\sin k_{e}\left(l_{n}-\left|x-x_{n}\right|\right)}{\sin k_{e} h},
\end{gathered}
$$




$$
\left|x-x_{n}\right|<l_{n},\left|y-y_{n}\right|<w_{i} / 2
$$

Where $l_{n}$ is the half-length of the expansion mode, $x_{n}$ and $y_{n}$ are the coordinates of the center of mode $n, w_{i}$ is the width of the strip to which the mode belongs, and $k_{e}$ is chosen as $k_{e}=k_{0} \sqrt{\left(\varepsilon_{r}+1\right) / 2}$. Eq. (1) is then solved using a Galerkin moment method solution, leading to the following equation:

$$
[Z][I]=[V]
$$

where $[z]$ is the impedance matrix of the antenna with elements given by:

$$
Z_{m n}=-\frac{1}{4 \pi^{2}} \int_{-\infty}^{\infty} \int_{-\infty}^{\infty} G_{x x}\left(k_{x}, k_{y}\right) F_{m}^{*}\left(k_{x}, k_{y}\right) F_{n}\left(k_{x}, k_{y}\right) d k_{x} d k_{y},
$$

$F_{n}\left(k_{x}, k_{y}\right)$ is the Fourier transform of the expansion mode $n$

$$
F_{n}\left(k_{x}, k_{y}\right)=\int_{x_{n}-l_{n}}^{x_{n}+l_{n}} \int_{y_{n}-w / 2}^{y_{n}+w / 2} f_{n}(x, y) e^{-j k_{x} x} e^{-j k_{y} y} d x d y,
$$

and * denotes the complex conjugate. The elements of the excitation voltage matrix $[V]$ are all zero except the elements corresponding to the generator locations.

The impedance matrix elements are obtained through numerical integration. The residues at the Green's function poles are computed with pole singularities extracted before the integration. These poles correspond to the zeros of Eqs. (2) and (3), that correspond to the characteristic equations for the $T M$ and $T E$ surface-wave modes of the ungrounded dielectric substrate [16]. There is always an amount of power coupled into these surface-waves because both the first $T M$ and $T E$ modes have zero cutoff frequencies. As the substrate thickness increases, the power coupled to the first $T E$ surface-wave mode increases rapidly [9], so strip dipole antennas are efficient only if printed on electrically thin dielectric substrates. Nevertheless, the amount of power coupled to surface-waves can be determined using the described method.

For planar antennas analyzed using the spectral domain approach, a stationary phase method is usually used to obtain the radiation patterns $[10,13]$. This method gives convenient and useful results except for points close to the dielectric substrate. In fact, patterns calculated in this way appear to have nulls at the air-dielectric interface since the substrate is assumed to be infinite. In practice, the H-plane patterns of strips printed on electrically thin ungrounded dielectric substrates of finite size show no nulls there. If strips are not printed close to the dielectric edge, the strip currents obtained via Eq. (7) would be accurate even though the dielectric is of infinite size, and accurate radiation patterns are obtained by assuming the strips radiate into free space. In this way the contribution of the polarization currents is not taken into account, but they are negligible for electrically thin dielectric substrates [17].

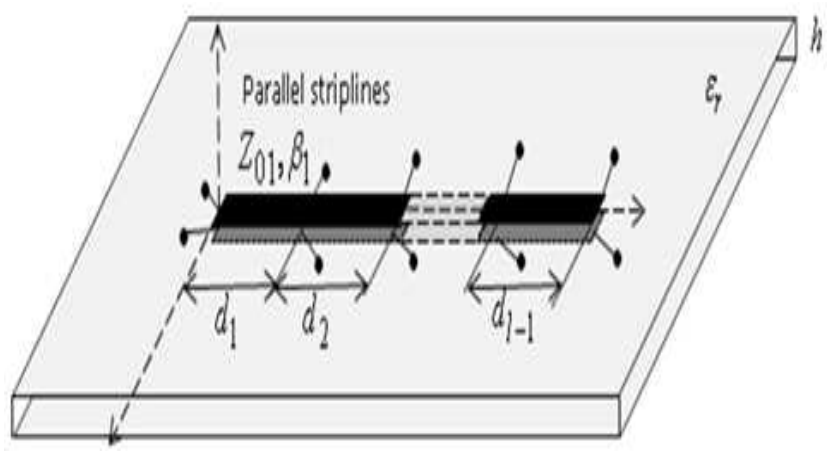

Figure3. Geometry of a group of $(l-1)$ connecting printed stripline.

To include the effect of the connecting line, the complete antenna system is considered as a parallel connection of two $l$-terminal networks, which can be represented by their admittance matrixes. One $l$-terminal network, $\left[Y_{A}\right]$, represents radiating elements with self and mutual admittances of Fig. 2, which are obtained by using the presented approach. The other $l$-terminal network, $\left[Y_{L}\right]$, represents the connecting lines with terminals at places

\begin{tabular}{|c|c|c|c|c|c|}
\hline \multirow{6}{*}[Y_{L}]{$=$} & $\left(-Y_{01} \cot \beta_{1} d_{1}\right.$ & $\pm j Y_{01} \csc \beta_{1} d_{1}$ & \multicolumn{3}{|c|}{0} \\
\hline & $\pm j Y \csc \beta_{1} d_{1}$ & $-j Y_{01} \cot \beta_{1} d_{1}-j Y_{02} \cot \beta_{2} d_{2}$ & \multicolumn{3}{|c|}{ $\pm j Y_{02} \csc \beta_{2} d_{2}$} \\
\hline & 0 & $-j Y_{02} \csc \beta_{2} d_{2}$ & $-j Y_{02}$ & $\cot \beta_{2} d_{2}-j Y_{03} \cot \beta_{3} d_{3}$ & $\ldots$ \\
\hline & $\ldots$ & $\ldots$ & & $\ldots$ & $\ldots$ \\
\hline & 0 & 0 & & 0 & $\ldots$ \\
\hline & 0 & 0 & & 0 & $\ldots$ \\
\hline$\ldots$ & \multicolumn{2}{|r|}{0} & \multicolumn{3}{|c|}{0} \\
\hline$\ldots$ & \multicolumn{2}{|r|}{0} & \multicolumn{3}{|c|}{0} \\
\hline$\ldots$ & \multicolumn{2}{|r|}{0} & \multicolumn{3}{|c|}{0} \\
\hline$\cdots$ & \multicolumn{2}{|c|}{$\ldots$} & \multicolumn{3}{|c|}{$\ldots$} \\
\hline$\cdots$ & \multicolumn{2}{|c|}{$-j Y_{0(l-2)} \beta_{(l-2)} d_{(l-2)}-j Y_{0(l-1)} \beta_{(l-1)} d_{(l-1}$} & \multicolumn{3}{|c|}{ $\pm j Y_{0(l-1)} \csc \beta_{(l-1-1} d_{(l-1)}$} \\
\hline$\cdots$ & \multicolumn{2}{|c|}{ $\pm j Y_{0(l-1)} \csc \beta_{(l-1)} d_{(l-1)}$} & \multicolumn{3}{|c|}{$-j Y_{0(l-1)} \cot \beta_{(l-1)} d_{(l-1)}$} \\
\hline
\end{tabular}
where strip dipoles are connected as shown in Fig. 3. The elements of the $\left[Y_{L}\right]$ matrix are determined using standard transmission line equations (see next equation):

$Z_{0 i}=1 / Y_{0 i}, \beta_{i}$, and $d_{i}, i=1,2, \ldots l$ are the characteristic impedance, the propagation constant, and the length, respectively, of the connecting line sections between strip dipoles. If the line polarity between the strip dipoles is notreversed (reversed), the plus (minus) sign must be used for off-diagonal elements. The $l$-port networks, $\left[Y_{A}\right]$ and $\left[Y_{L}\right]$, are connected in parallel as shown in Fig. 4. The overall $l$ port network as shown in Fig. 1 has $(l-1)$ ports open circuited, so that the input impedance at the first port can be readily obtained, and hence the performance of the antenna. The equivalent admittance matrix is also used to find the complex voltage of delta gap generators that are used to derive radiation characteristic of the antenna. In general not all printed dipoles have to be connected with transmission lines. Some of them can be made parasitic to form for example Yagi-Uda arrays of printed dipoles. 


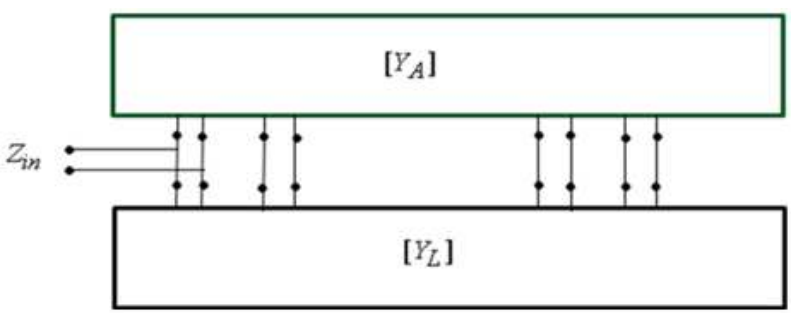

Figure4. Representation of the array of printed strip dipoles and the connecting lines with admittance matrices.

\section{Analysis Results of Arrays of Printed Strip Dipole Antennas}

In this section, analysis results for a series-fed array of two printed strip dipoles that operates as a dual-frequency antenna are presented. First, the effect of dielectric substrate on the input impedance of this antenna is shown in Fig.5 It can be seen that the calculated results agree well with experiments when the dielectric substrate is taken into account.

Antenna dimensions are like below:

Strip width: $w_{1}=w_{2}=6 \mathrm{~mm}$

Dipole lengths: $L_{1}=134 \mathrm{~mm}, L_{2}=78 \mathrm{~mm}$

Distance between dipoles: $d=50 \mathrm{~mm}$

Dielectric substrate thickness: $h=1.6 \mathrm{~mm}$

Dielectric substrate permittivity: $\varepsilon_{r}=3.2$

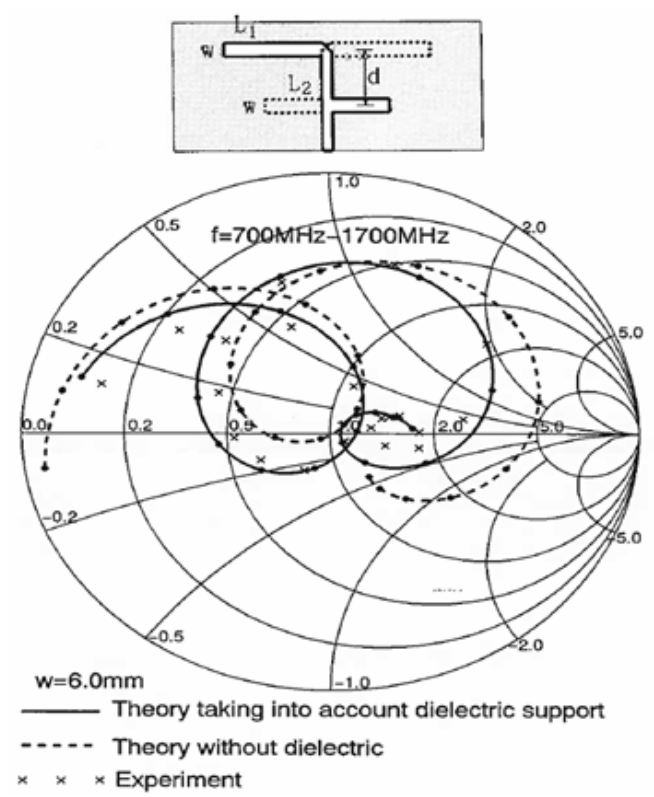

Figure5. Calculated and measured input impedance of the series-fed array of two printed strip dipoles

The dual-frequency operation characteristics of this antenna can be explained by observing the current distribution on each strip dipole at the two operating frequencies. At the first frequency of operation, $(0.9 \mathrm{GHz})$, the first dipole mainly contributes to radiation (see Fig 6-a), whereas in the second frequency of operation $(1.55 \mathrm{GHz})$, the second dipole mainly contributes to radiation (see Fig 6-b).

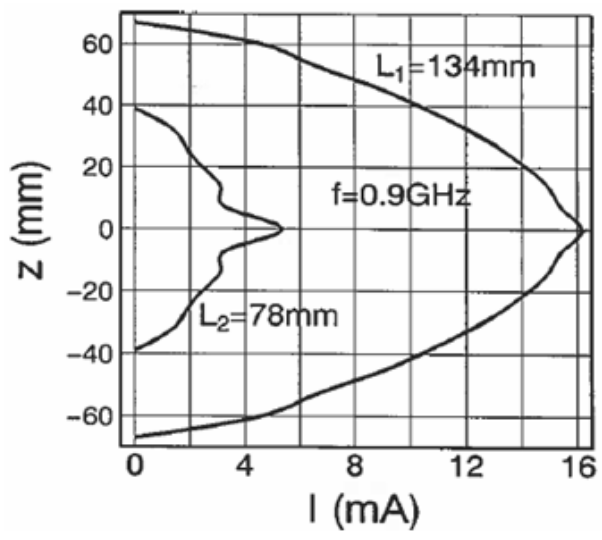

a) Distribution of each strip dipole at $0.9 \mathrm{GHz}$

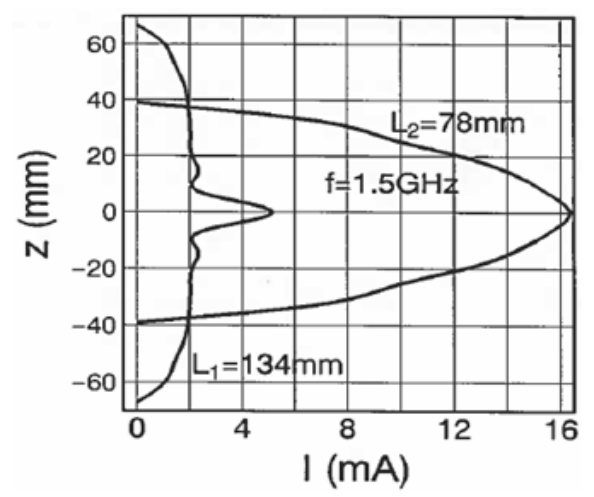

b) Distribution of each strip dipole at $1.55 \mathrm{GHz}$

Figure6. Calculated current amplitudes on the two strips of dualfrequency antenna.

We also analyzed the effect of the dielectric substrate on the radiation characteristics of series-fed strip dipoles and present a series fed array antenna of six-printed dipoles featuring broadband operation. This simple, easily manufactured antenna is well suited for the base station antennas of public mobile communication systems. Figure 7 shows a schematic diagram of the array antenna and input return loss of this antenna.

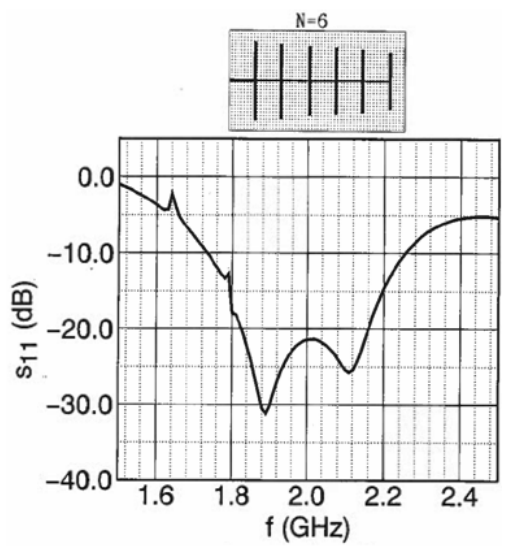

Figure7. Return loss of the series-fed array of six printed strip dipoles 
The end-fire characteristics of this antenna are shown in Fig. 8, where the relative front and back lobe levels and antenna gain against frequency are plotted.

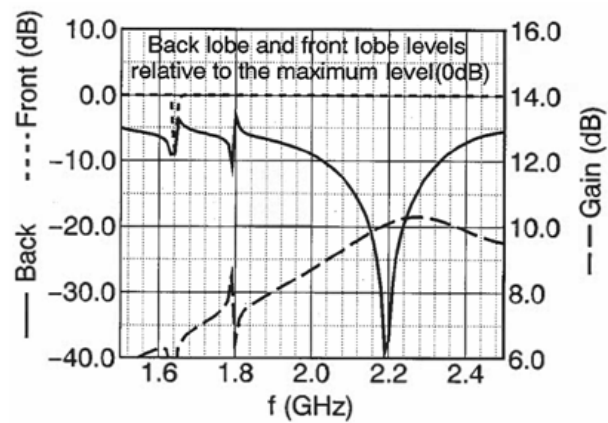

Figure8. Relative front and back lobe levels and gain of the series-fed array of six printed dipoles.

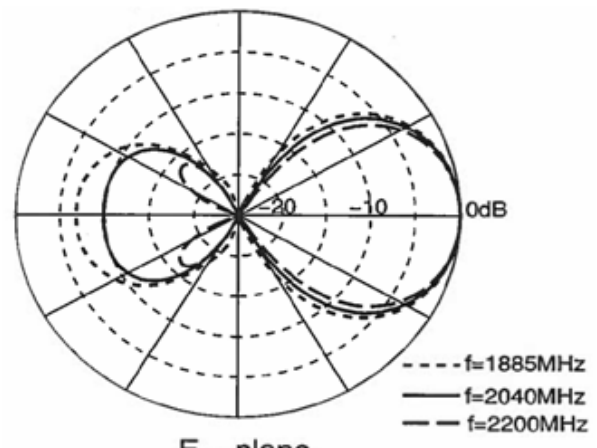

E - plane

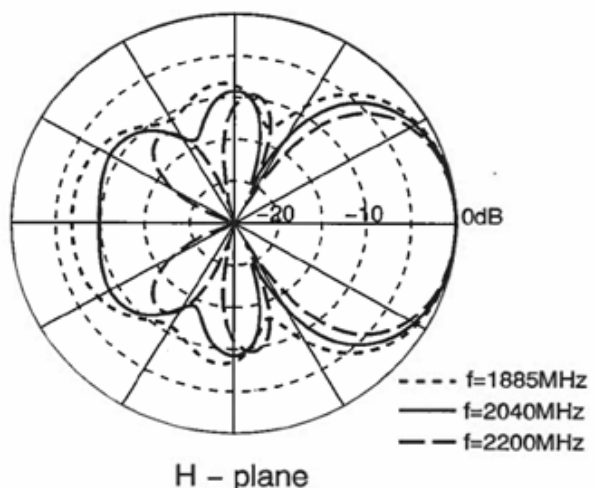

$\mathrm{H}$ - plane

Figure9. E- and H-plane radiation pattern of the series-fed array of six printed strip dipoles.

The calculated radiation patterns at two principal planes at frequencies $1885 \mathrm{MHz}, 2040 \mathrm{MHz}$ and $2200 \mathrm{MHz}$ are plotted in Fig. 9. Small variations on beamwidth of both E- and H-plans can be observed, showing broadband radiation characteristics of these antennas.

\section{Analysis and Results of Broadband Antenna of Six Series Fed Strip Dipoles}

Wireless technology in general is developing more and more. Increasing demand for high data rate transmission through wireless technology has urged implementation of broadband antennas for fulfilling these requirements with low cost. These wireless technologies are operating in multiple frequency bands. So, the idea is to use only one antenna that radiates in multiple frequencies. The benefits of using only one antenna are multifold, but here we will mention the cost reduction and the space.

In this section, first, we have analyzed a case of seriesfed arrays of six printed strip dipoles in front of plane reflector. The dimensions are: Distance between dipoles $\mathrm{D}=160 \mathrm{~mm}$ and dielectric substrate thickness $\mathrm{h}=30 \mathrm{~mm}$.
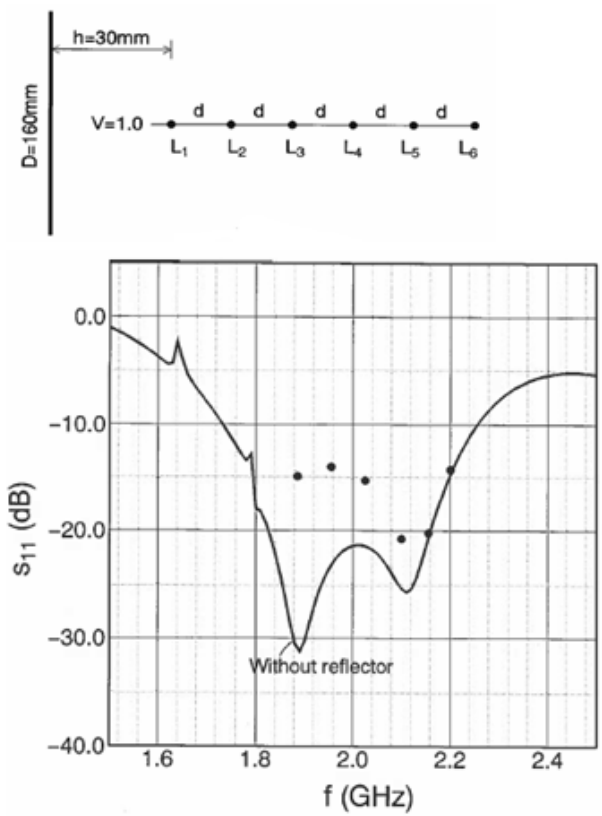

a) Calculated return loss

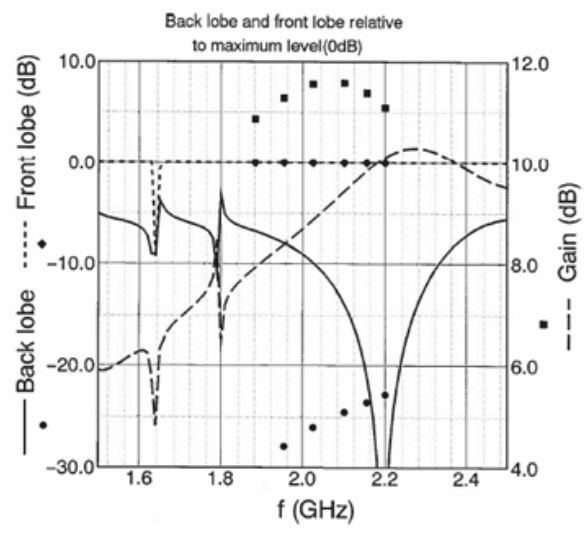

b) Calculated radiation characteristics

Figure10. a) Calculated return loss and b) Calculated radiation characteristics

In this case we have calculated only six frequencies. In this case it is used reflector calculation which is very intensive calculation. In the figure 10, we have presented calculated results of return loss (S11 parameters), for different frequencies.

We also analyzed the effect of the dielectric substrate on the radiation characteristics of series-fed arrays of six 
printed strip dipoles in front of plane reflector [18]. This simple, easily manufactured antenna is well suited for the base station antennas of public mobile communication systems. Figure 7 shows a schematic diagram of the array antenna and input return loss of this antenna.

Whereas, $\mathrm{H}$ plane radiations pattern are shown in the figure below:

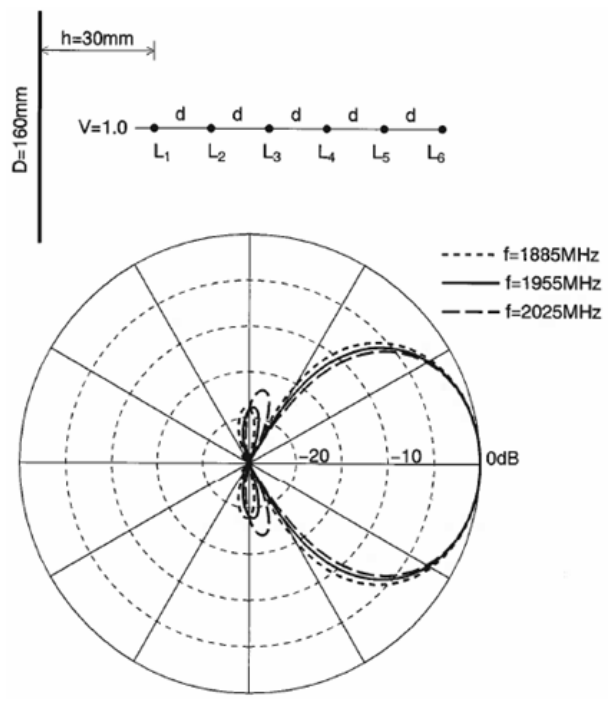

a)

H-plane radiation pattern $\mathrm{f}=1885 \mathrm{Mhz}, \mathrm{f}=1955 \mathrm{Mhz}$, and $\mathrm{f}=2025 \mathrm{Mhz}$

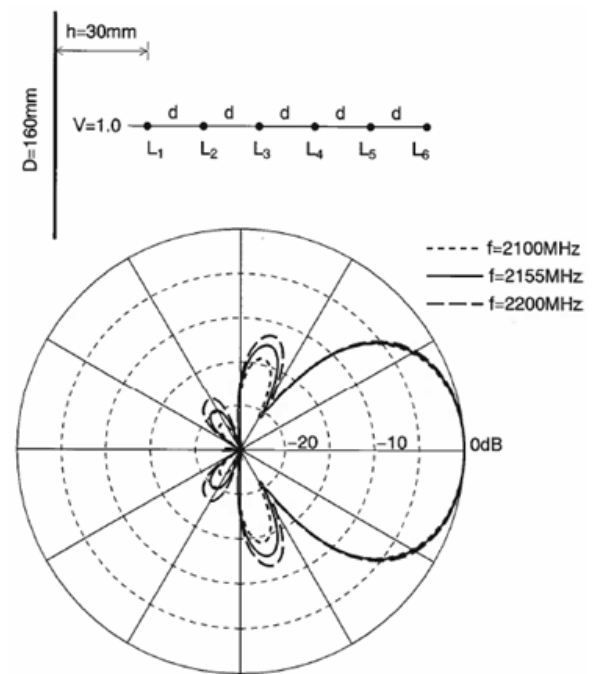

b) $\mathrm{H}$ plane radiation pattern for $\mathrm{f}=2100 \mathrm{Mhz}, \mathrm{f}=2155 \mathrm{Mhz}$, and $\mathrm{f}=2200 \mathrm{Mhz}$

Figure11. H plane radiation pattern for different frequencies

From figure 11, small variations on beamwidth of $\mathrm{H}$ plans can be observed, showing broadband radiation characteristics of these antennas.

Second case scenario: Two parallel arrays of series-fed printed strip dipole in front of a plane reflector. Only six frequencies calculated with reflector. Lines are for the same case without reflector for comparison

The end-fire characteristics of this antenna are shown in Fig. 12, where the relative front and back lobe levels and antenna gain against frequency are plotted.

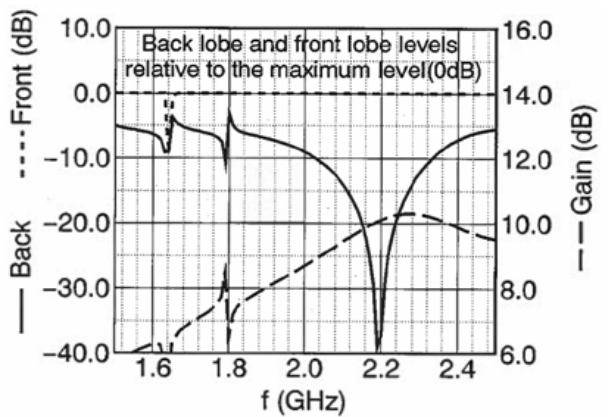

Figure12. Relative front and back lobe levels and gain of the two parallel arrays of series-fed strip dipoles

Figure 13 shows a schematic diagram of the two parallel of series - fed printed strip dipoles as well as a input return loss of this antenna's system.
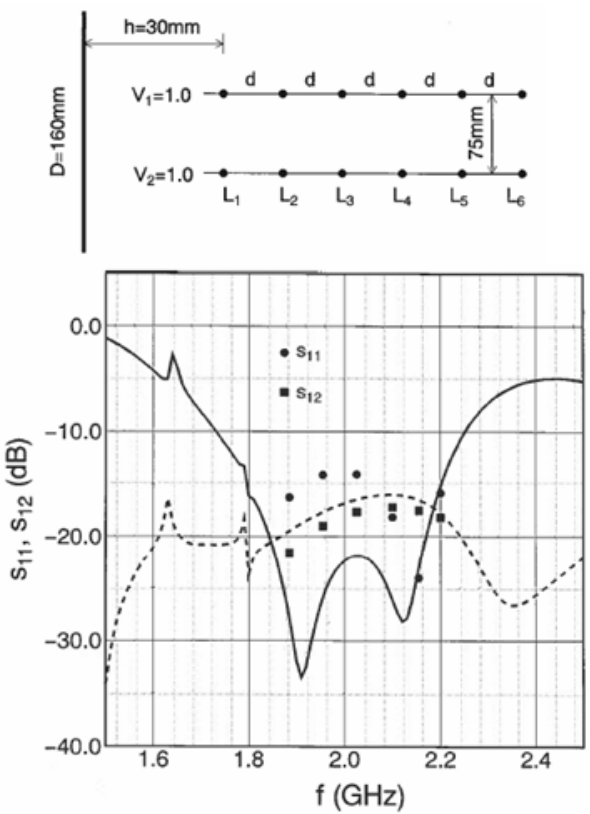

a) Calculated return loss

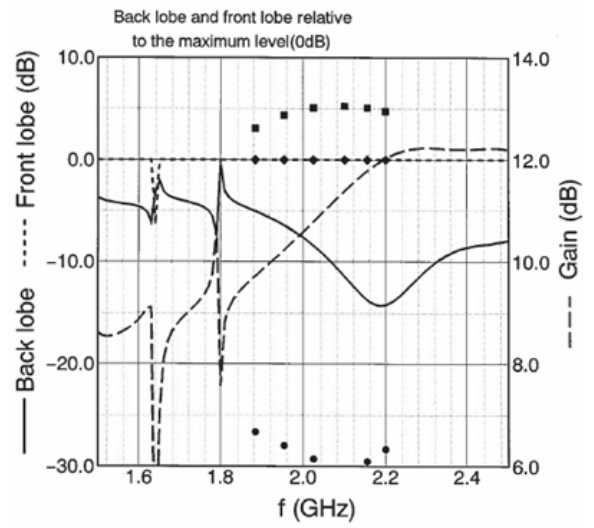

b) Calculated radiation characteristics

Figure13. a) Calculated return loss and b) Calculated radiation characteristics 
The calculated radiation patterns at frequencies 1885 $\mathrm{MHz}, \mathrm{f}=1955 \mathrm{MHz}$, and $\mathrm{f}=2025 \mathrm{Mhz}$ are plotted in Fig. 14.a. Whereas, the calculated radiation patterns at frequencies $\mathrm{f}=2100 \mathrm{MHz}, \mathrm{f}=2155 \mathrm{MHz}$, and $\mathrm{f}=2200 \mathrm{MHz}$ are plotted in Fig. 14.b.

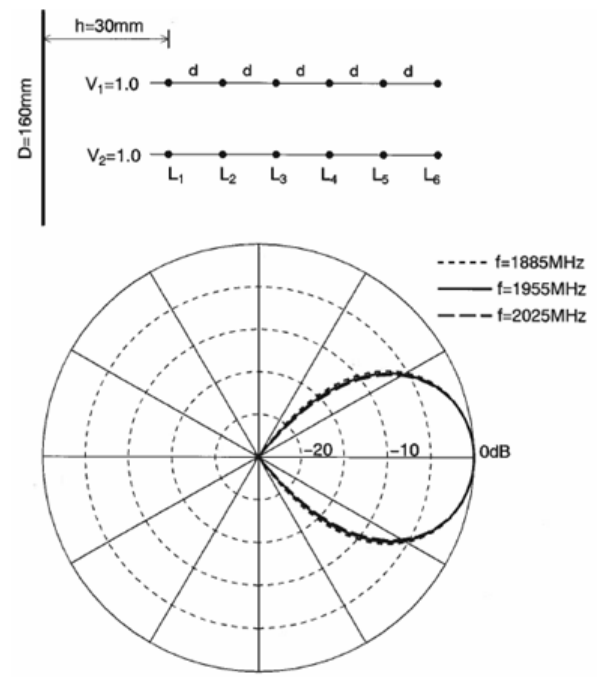

a)

H-plane radiation pattern $\mathrm{f}=1885 \mathrm{Mhz}, \mathrm{f}=1955 \mathrm{Mhz}$, and $\mathrm{f}=2025 \mathrm{Mhz}$
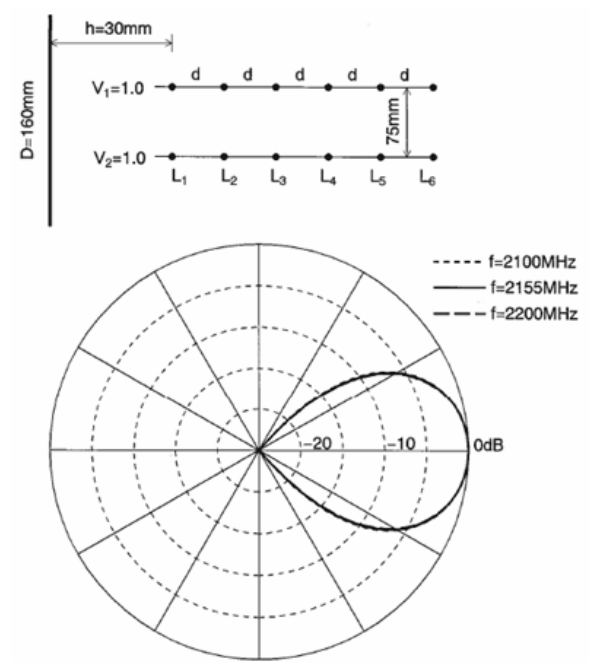

b) $\mathrm{H}$ plane radiation pattern for $\mathrm{f}=2100 \mathrm{Mhz}, \mathrm{f}=2155 \mathrm{Mhz}$, and $\mathrm{f}=2200 \mathrm{Mhz}$

Figure14. $H$ plane radiations pattern for a) $f=1885 \mathrm{MHz}, f=1955 \mathrm{Mhz}$, and $f=2025 \mathrm{Mhz}$ and $b) f=2100 \mathrm{Mhz}, f=2155 \mathrm{Mhz}$, and $f=2200 \mathrm{Mhz}$

In figure 14 , it can be observed variations on beamwidth of H-plans, showing broadband radiation characteristics of these antennas. As we can see, in this figure $x 4$.a, there is a very small differentiation on beamwidth for different frequencies, whereas in fig $x .4 b$, it is hard to see any difference for different frequencies.

\section{Summary}

In this paper have been analyzed different scenarios of printed strip dipole antennas. These analyses are done based on moment method in spectral domain. First, we have analyzed a case of series-fed arrays of six printed strip dipoles in front of plane reflector. In this case we have calculated only six frequencies. In this case it is used reflector calculation which is very intensive calculation.

Also we have analyzed two parallel arrays of series-fed printed strip dipole in front of a plane reflector. Only six frequencies calculated with reflector. Lines are for the same case without reflector for comparison. For this case, we have presented radiation patterns at frequencies 1885 $\mathrm{MHz}, \mathrm{f}=1955 \mathrm{MHz}, \mathrm{f}=2025 \mathrm{MHz}, \mathrm{f}=2100 \mathrm{MHz}, \mathrm{f}=2155$ $\mathrm{MHz}$, and $\mathrm{f}=2200 \mathrm{MHz}$. From this case, we can see observed variations on beamwidth of H-plans, showing broadband radiation characteristics of these antennas.

In this paper have been analyzed arrays of printed strip dipole antennas. As in [18], here also it is used moment method in the spectral domain. The double-sided configuration was selected because it offers several practical advantages such as: when required the line polarity between the strip dipoles can be easily reversed. To simplify the analysis, it was assumed that the conductive strips are printed on an infinite dielectric substrate and the effect of the connecting line is accounted for separately by using a standard transmission line theory. In this paper we also analyzed the effect of the dielectric substrate on the radiation characteristics of series-fed strip dipoles and present a series fed array antenna of six-printed dipoles featuring broadband operation. We have also analyzed different scenarios of printed strip dipole antennas. First, we have analyzed a case of series-fed arrays of six printed strip dipoles in front of plane reflector. In this case we have calculated only six frequencies. In this case it is used reflector calculation which is very intensive calculation.

Also we have analyzed two parallel arrays of series-fed printed strip dipole in front of a plane reflector. Only six frequencies calculated with reflector. Lines are for the same case without reflector for comparison. For this case, we have presented radiation patterns at frequencies 1885 $\mathrm{MHz}, \mathrm{f}=1955 \mathrm{MHz}, \mathrm{f}=2025 \mathrm{MHz}, \mathrm{f}=2100 \mathrm{MHz}, \mathrm{f}=2155$ $\mathrm{MHz}$, and $\mathrm{f}=2200 \mathrm{MHz}$. From this case, we can see observed variations on beamwidth of H-plans, showing broadband radiation characteristics of these antennas.

\section{References}

[1] K. Fujimoto and J. R. James, Mobile Antenna System Handbook, Artech House, Boston, 1994, ch. 3.

[2] F. Tefiku and C. A. Grimes, "Design of broadband and dualband antennas comprised of series-fed printed-strip dipole pairs," IEEE Trans. Antennas Propagat., vol. 48, pp. 895900, Jun. 2000.

[3] F. Tefiku and E. Yamashita, "Double-sided printed strip antenna for dual-frequency operation," IEEE AP-S Int. Symp. Dig., pp. 50-53, July 1996.

[4] F. Tefiku, "A broadband antenna of double-sided printed strip dipoles," Int. Symp. on Antennas and Propagat., Japan (ISAP'96), pp. 361-364, Sep. 1996. 
[5] F. Tefiku, "Broadband sector zone base station antennas," IEEE AP-S Conf. on Antennas and Propagat. for Wireless Communications, pp. 109-112, Nov. 1998.

[6] A. J. Parfitt, D. W. Griffin, and P. H. Cole, "Analysis of infinite arrays of substrate-supported metal strip antennas," IEEE Trans. Antennas Propagat., vol. 41, pp. 191-199, Feb. 1993.

[7] J. R. Bayard, M. E. Cooley, and D.H. Schaubert, "Analysis of infinite arrays of printed dipoles on dielectric sheet perpendicular to a ground plane," IEEE Trans. Antennas Propagat., vol. 39, pp. 1722-1732, Dec. 1991.

[8] B. Edward and D. Rees, "A broadband printed dipole with integrated balun,” Microwave J., pp. 339-344, May 1987.

[9] D. B. Rutledge, D. P. Neikirk, and D. P. Kasilingam, "Integrated -circuit antennas," in Infrared and Millimeter Waves, vol.10, pp. 1-91, K. J. Button, Ed., New York: Academic, 1983.

[10] M. Kominami, D. M. Pozar, and D. H. Schaubert, "Dipole and slot elements and arrays on semi-infinite substrates," IEEE Trans. Antennas Propagat., vol. 33, pp. 600-607, June 1995.

[11] A. K. Agrawal and W. E. Powell, "A printed circuit cylindrical array antenna," IEEE Trans. Antennas Propagat., vol. 34, pp. 1288-1293, Nov.1986.
[12] E. Levine, S. Shtrikman, and D. Treves, "Double-sided printed arrays with large bandwidth," IEE Proc. H, Microwave, Opt. \& Antennas, vol. 135, pp. 54-59, Feb 1988.

[13] D. M. Pozar, "Analysis of finite phased arrays of printed dipoles," IEEE Trans. Antennas Propagat., vol. 33, pp. 1045-1053, Oct.1985.

[14] R. Sefa, A. Maraj, "Design of microstrip to balanced striplines transitions" The 10th WSEAS International Conference on TELECOMMUNICATIONS and INFORMATICS (TELE-INFO '11), Included in ISI/SCI Web of Science and Web of Knowledge.

[15] I. Tekin and E. H. Newman, "Space-domain method of moments solution for a strip on a dielectric slab," IEEE Trans. Antennas Propagat., vol. 46, pp. 1346-1348, Sep.1998.

[16] R. F. Harrington, Time Harmonic Electromagnetic Fields, McGraw-Hill, New York, 1961, chap. 4.

[17] R. Janaswamy, "An accurate moment method model for the tapered slot antenna," IEEE Trans. Antennas Propagat., vol. 37, pp. 1523-1528, Dec. 1989.

[18] R. Sefa, F. Tefiku, A. Maraj, "Analysis of arrays of printed strip dipole antennas" Software, Telecommunications and Computer Networks (SoftCOM), 2011 19th International Conference, Date of Conference: 15-17 Sept, IEEE Conference, 2011 